TITLE:

\title{
Singular behaviour of a rarefied gas on a planar boundary
}

$\operatorname{AUTHOR}(\mathrm{S})$ :

Takata, Shigeru; Funagane, Hitoshi

CITATION:

Takata, Shigeru ... [et al]. Singular behaviour of a rarefied gas on a planar boundary. Journal of Fluid Mechanics 2013, 717: 30-47

ISSUE DATE:

2013-02

URL:

http://hdl.handle.net/2433/193963

RIGHT:

(C)2013 Cambridge University Press 


\title{
Singular behaviour of a rarefied gas on a planar boundary
}

\author{
Shigeru Takata ${ }^{1,2, \dagger}$ and Hitoshi Funagane ${ }^{1}$ \\ ${ }^{1}$ Department of Mechanical Engineering and Science, Graduate School of Engineering, Kyoto University, \\ Kyoto 606-8501, Japan \\ ${ }^{2}$ Advanced Research Institute of Fluid Science and Engineering, Graduate School of Engineering, \\ Kyoto University, Kyoto 606-8501, Japan
}

(Received 9 April 2012; revised 10 September 2012; accepted 9 November 2012; first published online 1 February 2013)

Singular behaviour of a rarefied gas on a planar boundary is clarified on the basis of the Boltzmann equation. The thermal transpiration between two parallel plates is taken as a specific example. First, the flow velocity is shown to behave like $x \ln x$ in the vicinity of the boundary, where $x$ is a distance from the boundary. This implies a logarithmic divergence of the flow velocity gradient as $x \rightarrow 0$. Then, such a spatial singularity is shown to induce a similar singularity of the velocity distribution function (VDF) with respect to $\zeta_{n}$ on the boundary, where $\zeta_{n}$ is a normal component of the molecular velocity to the boundary. Moreover, the spatial singularity is shown to be quantitatively related to the discontinuity of the VDF on the boundary at $\zeta_{n}=0$. These macroscopic and microscopic singularities should be observed generally in a rarefied gas on a planar boundary.

Key words: kinetic theory, non-continuum effects, rarefied gas flow

\section{Introduction}

The steady behaviour of a rarefied gas bounded by a planar wall is a fundamental boundary-value problem in the kinetic theory of gases and has been studied extensively from theoretical and practical interests. The behaviour of a rarefied gas is usually investigated by using the Boltzmann equation or its model equation such as the Bhatnagar-Gross-Krook (BGK or Boltzmann-Krook-Welander (BKW)) model (Bhatnagar, Gross \& Krook 1954; Welander 1954) and the ellipsoidal statistical (ES) model (Holway 1963, 1966). In the present paper, on the basis of the Boltzmann equation, we will first show that macroscopic quantities defined as a moment of the velocity distribution function (VDF) behave singularly on a planar boundary.

To be more specific, if denoting by $x$ the distance from the planar boundary, the singular behaviour mentioned above means that the macroscopic quantities vary in $x$ in the vicinity of the boundary as $x \ln x$ and thus their gradient diverges logarithmically as $x \rightarrow 0$. Such behaviour on the boundary has already been pointed out as early as the 1960s and 1970s by Sone $(1964,1965)$ in the study of the long time behaviour of a gas in the Rayleigh problem and its similar problem and by Sone \& Onishi (1978) 
in the study of the Knudsen layer for a slightly rarefied gas (namely, for small Knudsen numbers) on the basis of the BGK model. Judging from their analyses, the logarithmic gradient divergence is expected to appear irrespective of the Knudsen number. However, a concrete analysis discussing this point has not been found in the literature until quite recently. Chen, Liu \& Takata (2010) proved mathematically the logarithmic divergence of the flow-velocity gradient of the thermal transpiration (Kennard 1938) between two parallel plates for large Knudsen numbers, by developing the mathematical and numerical analyses of Chen et al. (2007) and Takata \& Funagane (2011) for a hard-sphere gas. In the present paper, we will go a step further and first show in $\S 3$ that the same logarithmic divergence appears not only in the regimes of small and high Knudsen numbers but also in the regime of intermediate Knudsen numbers. Recently, Lilley \& Sader $(2007,2008)$ have also pointed out the gradient divergence of macroscopic quantities on the boundary. Their discussions are, however, based on finding a power law $x^{\alpha}(0<\alpha<1)$ that fits well to existing numerical data in the literature or newly computed data obtained by the direct simulation Monte Carlo (DSMC) method (Bird 1994). As a result, the logarithmic feature of divergence has not been clarified.

In the present paper, for the clarity and definite discussions, we shall exclusively study the thermal transpiration between two parallel plates (e.g. Maxwell 1879; Kennard 1938; Sone 1966, 2007; Loyalka 1971; Niimi 1971; Williams 1971; Ohwada, Sone \& Aoki 1989), assuming a hard-sphere gas and the diffuse reflection boundary condition. However, the singular features to be clarified here should be observed on a planar boundary, irrespective of the Knudsen number, for more general intermolecular potential models and boundary conditions.

The macroscopic quantities are defined as a moment of the VDF in the molecular velocity space. Hence, their singularity $x \ln x$ near the boundary strongly suggests the same singular feature of the collision term. We will show in $\S 4$ that the spatial singularity of the collision integral gives rise to another singularity of the VDF on the boundary in the form of $\zeta_{n} \ln \left|\zeta_{n}\right|$ for $\zeta_{n} \sim 0_{-}$, where $\zeta_{n}$ is the normal component of the molecular velocity to the boundary, pointed to the gas. Thus, the derivative of the VDF in $\zeta_{n}$ exhibits the logarithmic divergence for the molecular velocities parallel to the boundary. This microscopic singularity is the second main result of the present paper that has not been pointed out in the literature. Moreover, we will show in $\S 5$ that the spatial singularity of the macroscopic quantities can be related quantitatively to the discontinuity of the VDF on the boundary at $\zeta_{n}=0$. This relation is the third main result of the present paper, which has also not been pointed out in the literature.

One may think the divergence of the flow velocity gradient unreasonable, because it means the divergence of the shear stress in the conventional fluid-dynamics. It should be noted, however, that the stress tensor in a rarefied gas is not expressed by the shear velocity. In fact, in the case of the thermal transpiration to be studied below, the stress tensor is diagonal and isotropic and never diverges. This statement applies even to the regime of small Knudsen numbers, in which the fluid-dynamic part of the flow velocity is uniform in space and its kinetic boundary-layer correction (non-fluiddynamic part or the Knudsen-layer correction) exhibits the gradient divergence on the boundary.

\section{Problem}

As mentioned in $\S 1$, we consider the thermal transpiration of a rarefied gas between two parallel plates as a specific problem. 


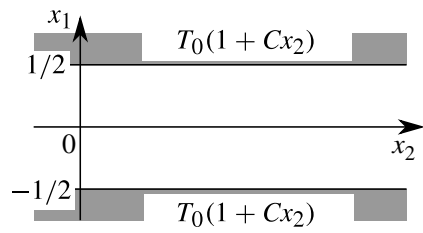

FIGURE 1. Thermal transpiration.

Consider a rarefied gas between two parallel walls which are separated by $D$. Let us denote the spatial coordinates by $D \boldsymbol{x}$, where the $x_{1}$ direction is normal to the walls, while the $x_{2}$ and $x_{3}$ directions are parallel to the walls (figure 1). We will study the steady gas behaviour in the case that the wall temperature changes linearly in $x_{2}$ and given by $T_{0}\left(1+C x_{2}\right)$ (where $T_{0}$ is the reference temperature and $C$ is a constant) under the following assumptions.

(a) The behaviour of the gas is described by the Boltzmann equation (or its model kinetic equation).

(b) The gas molecules are diffusely reflected on the walls.

(c) The value of $|C|$ is so small that the equation and boundary condition can be linearized around the reference equilibrium state at rest with density $\rho_{0}$ (the average density) and temperature $T_{0}$.

Denoting the specific gas constant by $R$, the molecular velocity by $\left(2 R T_{0}\right)^{1 / 2} \zeta$ and the velocity distribution function by $\rho_{0}\left(2 R T_{0}\right)^{-3 / 2}(1+\Phi) E(|\zeta|)$, where $E(|\zeta|)=\pi^{-3 / 2} \exp \left(-|\zeta|^{2}\right)$, the problem is described by the following equation and boundary condition (see, e.g., Sone (2007) for the standard process of the linearization):

$$
\left.\begin{array}{c}
\zeta_{1} \frac{\partial \Phi}{\partial x_{1}}+\zeta_{2} \frac{\partial \Phi}{\partial x_{2}}=-\frac{1}{k} v(|\zeta|) \Phi+\frac{1}{k} K[\Phi], \\
\text { b.c. } \Phi=\left(|\zeta|^{2}-2\right) C x_{2}+2 \sqrt{\pi} \int_{\zeta_{1} \gtrless 0}\left|\zeta_{1}\right| \Phi E(|\zeta|) \mathrm{d} \zeta, \\
\zeta_{1} \lessgtr 0, x_{1}= \pm \frac{1}{2} .
\end{array}\right\}
$$

Here $\mathrm{d} \zeta=\mathrm{d} \zeta_{1} \mathrm{~d} \zeta_{2} \mathrm{~d} \zeta_{3}, k=(\sqrt{\pi} / 2)\left(\ell_{0} / D\right)$, where $\ell_{0}$ is the mean free path of a gas molecule at the reference equilibrium state, and $k(0<k<\infty)$ is a parameter of the degree of gas rarefaction rate $(k \rightarrow 0$ is the continuum or fluid-dynamic limit, while $k \rightarrow \infty$ is the collisionless or free molecular limit). Note that the linearized collision operator $\mathscr{L}$ is composed of $-v$ and $K: \mathscr{L}[\Phi]=-v \Phi+K[\Phi]$. In the case of a hard-sphere gas, $v$ and $K$ are expressed as

$$
\begin{gathered}
\nu(|\zeta|)=\frac{1}{2 \sqrt{2}}\left(\exp \left(-|\zeta|^{2}\right)+\left(2|\zeta|+\frac{1}{|\zeta|}\right) \int_{0}^{|\zeta|} \exp \left(-t^{2}\right) \mathrm{d} t\right) \\
K[\Phi]=\int \kappa(\xi, \zeta) \Phi(\xi) E(|\xi|) \mathrm{d} \xi \\
\kappa(\xi, \zeta)=\frac{\sqrt{\pi}}{\sqrt{2}} \frac{1}{|\xi-\zeta|} \exp \left(\frac{|\xi \times \zeta|^{2}}{|\xi-\zeta|^{2}}\right)-\frac{\sqrt{\pi}}{2 \sqrt{2}}|\xi-\zeta|
\end{gathered}
$$


while, in the case of the BGK model, they are expressed as

$$
v(|\zeta|)=1, \quad K[\Phi]=P[\Phi]+2 \zeta_{i} u_{i}[\Phi]+\left(|\zeta|^{2}-\frac{5}{2}\right) \tau[\Phi]
$$

where

$$
\left.\begin{array}{l}
P[\Phi]=\frac{2}{3} \int|\zeta|^{2} \Phi E(|\zeta|) \mathrm{d} \zeta, \quad u_{i}[\Phi]=\int \zeta_{i} \Phi E(|\zeta|) \mathrm{d} \zeta, \\
\tau[\Phi]=\frac{2}{3} \int\left(|\zeta|^{2}-\frac{3}{2}\right) \Phi E(|\zeta|) \mathrm{d} \zeta,
\end{array}\right\}
$$

and $\rho_{0} R T_{0}(1+P[\Phi]),\left(2 R T_{0}\right)^{1 / 2} u_{i}[\Phi]$ and $T_{0}(1+\tau[\Phi])$ are the pressure, flow velocity and temperature of the gas, respectively. In the meantime, the stress tensor is denoted by $\rho_{0} R T_{0}\left(\delta_{i j}+P_{i j}[\Phi]\right)$, where $\delta_{i j}$ is Kronecker's delta, in terms of

$$
P_{i j}[\Phi]=2 \int \zeta_{i} \zeta_{j} \Phi E(|\zeta|) \mathrm{d} \zeta
$$

The solution $\Phi\left(x_{1}, x_{2}, \zeta\right)$ can be sought in the form of

$$
\Phi=C\left[x_{2}\left(|\zeta|^{2}-\frac{5}{2}\right)+\phi\left(x_{1}, \zeta\right)\right]
$$

where $\phi$ is a solution of the following boundary-value problem that is odd in $\zeta_{2}$ and even in $\zeta_{3}$ :

$$
\begin{gathered}
\zeta_{1} \frac{\partial \phi}{\partial x_{1}}=-\frac{1}{k} v(|\zeta|) \phi+\frac{1}{k} K[\phi]-\zeta_{2}\left(|\zeta|^{2}-\frac{5}{2}\right), \\
\text { b.c. } \quad \phi=0, \quad \zeta_{1} \lessgtr 0, \quad x_{1}= \pm \frac{1}{2} .
\end{gathered}
$$

This problem can be solved formally as

$$
\phi=\int_{\mp 1 / 2}^{x_{1}} \frac{1}{k \zeta_{1}} \exp \left(-\frac{v(|\zeta|)}{k\left|\zeta_{1}\right|}\left|x_{1}-s\right|\right) K[\phi](s, \zeta) \mathrm{d} s+\phi_{0}, \quad \zeta_{1} \gtrless 0,
$$

where

$$
\begin{gathered}
\phi_{0}=-\frac{k}{v(|\zeta|)}\left[1-\exp \left(-\frac{v(|\zeta|)}{k \zeta_{1}}\left(x_{1} \pm \frac{1}{2}\right)\right)\right] I, \quad \zeta_{1} \gtrless 0, \\
I=\zeta_{2}\left(|\zeta|^{2}-\frac{5}{2}\right) .
\end{gathered}
$$

Hereafter, the argument of $E$ and $v$ will be omitted, unless confusion is expected. In the course of analyses, another coordinate system $(\zeta, \mu, \varphi)$ is also used for the dimensionless molecular velocity $\zeta$ :

$$
\left.\begin{array}{l}
\zeta=|\zeta|, \quad \mu=\zeta_{1} /|\zeta|, \quad \tan \varphi=\zeta_{3} / \zeta_{2} \\
(0 \leqslant \zeta<\infty,-1 \leqslant \mu \leqslant 1,0 \leqslant \varphi<2 \pi)
\end{array}\right\}
$$

It should be noted that $\phi / \zeta_{2}$ is a function of three variables $\left(x_{1}, \zeta_{1},|\zeta|\right)$ or $\left(x_{1}, \mu, \zeta\right)$

Because $\phi$ is odd in $\zeta_{2}$ and even in $\zeta_{3}$, it is seen by a direct substitution of (2.8) that the flow can occur in the $x_{2}$ direction $\left(u_{1}[\Phi]=0\right.$ and $\left.u_{3}[\Phi]=0\right)$ with a uniform pressure $(P[\Phi]=0)$ and temperature gradient $\left(\tau[\Phi]=C x_{2}\right)$. Because of the simplicity of the field, $K[\phi]$ of the BGK model is reduced to $K[\phi]=2 \zeta_{2} u_{2}[\phi]$.

Furthermore, the direct substitution shows that the perturbation of the stress tensor $P_{i j}[\Phi]$ vanishes except for the component $P_{12}[\Phi]\left(=P_{21}[\Phi]\right)$. The $P_{12}[\Phi]$ is also seen 
to vanish by the momentum balance discussion in the $x_{2}$ direction at the macroscopic level as follows. As is well known, the Boltzmann equation gives the macroscopic momentum balance equation if it is integrated in the molecular velocity space after multiplied by the molecular velocity. If we integrate (2.1a) multiplied with $\zeta_{2} E(|\zeta|)$ over the whole space of $\zeta$, we obtain the linearized version of the macroscopic momentum balance equation, which reads $\partial P_{12}[\Phi] / \partial x_{1}+\partial P_{22}[\Phi] / \partial x_{2}=0$. Here we have used the fact that the contribution from the collision integral, namely that from the right-hand side of $(2.1 a)$, vanishes because of the conservation of the momentum during the intermolecular collision process. On the other hand, $P_{22}[\Phi]$ vanishes and $P_{12}[\Phi]$ is independent of $x_{2}$, because of (2.8). Therefore, the momentum balance equation shows that $P_{12}[\Phi]$ is a constant. (If starting from (2.9a), one obtains $\partial P_{12}[\phi] / \partial x_{1}=0$ and thus $P_{12}[\phi]$ is constant. Because of (2.8), $P_{12}[\Phi]$ is identical with $P_{12}[\phi]$, and thus $P_{12}[\Phi]$ is a constant.) Now, because of the symmetry of the problem, $\phi$ (and thus $\Phi$ ) is symmetric with respect to $\zeta_{1}$ at $x_{1}=0$, which concludes that $P_{12}[\Phi]$ is identically zero. Therefore, the stress tensor is diagonal and isotropic $\left(P_{i j}[\Phi]=0\right)$, as mentioned in the last paragraph of $\S 1$.

We close the present section with a brief physical explanation of the thermal transpiration flow for the interested reader, following Sone (2007). Here we assume the diffuse reflection condition on the wall, but the discussion is essentially the same for more general cases. (The specular reflection wall is excluded here, because it is adiabatic wall and thermal information is not transferred to the gas molecules from the wall. Accordingly the thermal transpiration flow does not occur.) Suppose that there is no flow in the channel whose wall temperature grows along its surface (the wall is hotter for larger $x_{2}$, see figure 1). In this situation, the mass flow of molecules with the positive $x_{2}$ component balances with that of molecules with the negative $x_{2}$ component. Now, consider a position, say $\mathrm{P}$, on the wall. The molecules coming from the hotter region bring the negative momentum in the $x_{2}$ direction to the position $\mathrm{P}$, while those from the colder region bring the positive momentum in the $x_{2}$ direction there. Because the velocity of molecules from the hotter region is larger on average than that from the colder region, the incoming molecules to the wall at the position $\mathrm{P}$ bring the negative tangential momentum in total. On the other hand, both groups of molecules are reflected isotropically and thus have no momentum on average in the $x_{2}$ direction after the reflection. These mean that the momentum transfer from the gas to the wall occurs in the opposite $x_{2}$ direction. Because the wall is fixed, the force in the $x_{2}$ direction acts on the gas by the law of action and its reaction, and the gas starts to flow in the $x_{2}$-direction. As the flow accelerated, the induced flow increases (or reduces) the positive (or negative) momentum transfer from the colder (or hotter) region to the position P. Finally, the acceleration ceases when the induced flow cancels out the momentum transfer from the gas to the wall, which also explains why the shear stress does not occur in the present problem. This makes a remarkable contrast to the case of the Poiseuille flow, where the non-zero shear stress occurs for balancing with the imposed pressure gradient.

\section{Singularity of macroscopic quantities}

\subsection{Perspective of analysis}

We consider the $x_{2}$ component of the dimensionless flow velocity $u_{2}[\phi]\left(=u_{2}[\Phi]\right)$. In order to show the essence of the origin of the spatial singularity, we first discuss the contribution from $\phi_{0}$ only, which corresponds to neglecting the contribution from $K[\phi]$ in the formal solution $(2.10 a)$. 
By a straightforward calculation, we have the following expression for $u_{2}\left[\phi_{0}\right]$ :

$$
\begin{aligned}
u_{2}\left[\phi_{0}\right]= & \int \zeta_{2} \phi_{0} E \mathrm{~d} \zeta \\
= & -\int_{\zeta_{1}>0} \frac{k}{v}\left[1-\exp \left(-\frac{v}{k \zeta_{1}}\left(x_{1}+\frac{1}{2}\right)\right)\right] \zeta_{2}^{2}\left(|\zeta|^{2}-\frac{5}{2}\right) E \mathrm{~d} \zeta \\
& -\int_{\zeta_{1}<0} \frac{k}{v}\left[1-\exp \left(-\frac{v}{k \zeta_{1}}\left(x_{1}-\frac{1}{2}\right)\right)\right] \zeta_{2}^{2}\left(|\zeta|^{2}-\frac{5}{2}\right) E \mathrm{~d} \zeta \\
= & -\pi \int_{0}^{\infty} \frac{k \zeta^{4}}{v(\zeta)}\left(\zeta^{2}-\frac{5}{2}\right) E(\zeta) \int_{0}^{1}\left[1-\exp \left(-\frac{v(\zeta)}{k \zeta \mu}\left(x_{1}+\frac{1}{2}\right)\right)\right] \\
& \times\left(1-\mu^{2}\right) \mathrm{d} \mu \mathrm{d} \zeta \\
& -\pi \int_{0}^{\infty} \frac{k \zeta^{4}}{v(\zeta)}\left(\zeta^{2}-\frac{5}{2}\right) E(\zeta) \int_{-1}^{0}\left[1-\exp \left(-\frac{v(\zeta)}{k \zeta \mu}\left(x_{1}-\frac{1}{2}\right)\right)\right] \\
& \times\left(1-\mu^{2}\right) \mathrm{d} \mu \mathrm{d} \zeta \\
= & \frac{k}{6 \sqrt{\pi}} \sum_{i=+,-} \int_{0}^{\infty} \frac{\zeta^{4}}{v(\zeta)}\left(\zeta^{2}-\frac{5}{2}\right)\left(a_{i}^{2}-6\right) a_{i} \operatorname{Ei}\left(1, a_{i}\right) \exp \left(-\zeta^{2}\right) \mathrm{d} \zeta \\
& +\frac{k}{6 \sqrt{\pi}} \sum_{i=+,-} \int_{0}^{\infty} \frac{\zeta^{4}}{v(\zeta)}\left(\zeta^{2}-\frac{5}{2}\right)\left(4+a_{i}-a_{i}^{2}\right) \exp \left(-a_{i}-\zeta^{2}\right) \mathrm{d} \zeta \\
& -\frac{4 k}{3 \sqrt{\pi}} \int_{0}^{\infty} \frac{\zeta^{4}}{v(\zeta)}\left(\zeta^{2}-\frac{5}{2}\right) \exp \left(-\zeta^{2}\right) \mathrm{d} \zeta,
\end{aligned}
$$

where

$$
a_{ \pm}=\frac{v(\zeta)}{k \zeta}\left|x_{1} \pm \frac{1}{2}\right|, \quad \operatorname{Ei}(1, a>0)=\int_{1}^{\infty} \frac{1}{x} \exp (-a x) \mathrm{d} x .
$$

Because there is a positive constant $C_{0}$ such that $v(\zeta) \geqslant C_{0}>0$, the second term $\int_{0}^{\infty} \zeta^{4} v^{-1}\left(\zeta^{2}-5 / 2\right)\left(4+a_{ \pm}-a_{ \pm}^{2}\right) \exp \left(-a_{ \pm}-\zeta^{2}\right) d \zeta$ does not show the singularity. On the other hand, as is seen from the series expression of the exponential integral

$$
\operatorname{Ei}(1, a>0)=-\gamma-\ln a+e^{-a} \sum_{n=1}^{\infty} \frac{1}{n !}\left(\sum_{r=1}^{\infty} \frac{1}{r}\right) a^{n}, \quad(\gamma: \text { Euler's constant }),
$$

the first term $\int_{0}^{\infty} \zeta^{4} v^{-1}\left(\zeta^{2}-5 / 2\right)\left(a_{ \pm}^{2}-6\right) a_{ \pm} \operatorname{Ei}\left(1, a_{ \pm}\right) \exp \left(-\zeta^{2}\right) \mathrm{d} \zeta$ has a component that is proportional to $\left(x_{1} \pm 1 / 2\right) \ln \left|x_{1} \pm 1 / 2\right|$. Hence, $u_{2}\left[\phi_{0}\right]$ behaves accordingly and its gradient $\mathrm{d} u_{2}\left[\phi_{0}\right] / \mathrm{d} x_{1}$ diverges logarithmically as $x_{1} \rightarrow \mp 1 / 2$.

The above calculation shows the spatial singularity of $u_{2}\left[\phi_{0}\right]$, not the singularity of $u_{2}[\phi]$. Nevertheless, it presents the essence of the latter. Indeed, Chen et al. (2010) have shown for sufficiently large $k$ that the magnitude of $u_{2}\left[\phi_{0}\right]$ is much larger than that of the remainder $u_{2}\left[\phi-\phi_{0}\right]$ originating from $K[\phi]$ and that $u_{2}\left[\phi-\phi_{0}\right]$ does not have a singularity stronger than $\left(x_{1} \pm 1 / 2\right) \ln \left|x_{1} \pm 1 / 2\right|$. In the present paper, we will numerically show the appearance of the same spatial singularity, irrespective of the magnitude of $k$.

\subsection{Numerical analysis}

In Takata \& Funagane (2011), for accurate numerical computations in the highly rarefied regime $(k \gg 1)$, we have adopted the following iterative approximation 

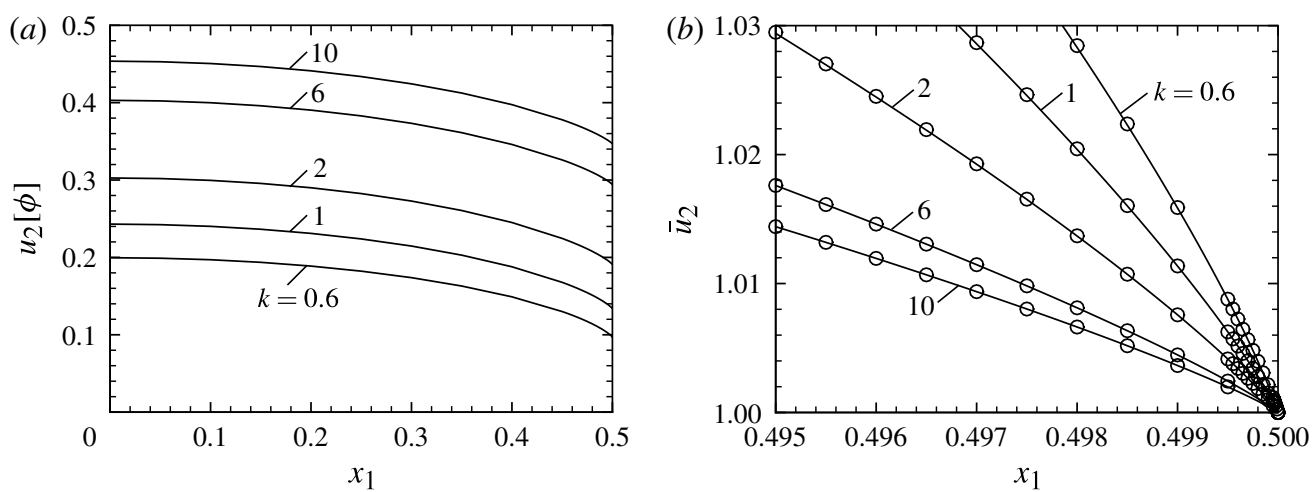

FIGURE 2. Profile of the flow velocity for various $k:(a) u_{2}[\phi]\left(x_{1}\right)$ in the half-channel $0 \leqslant x_{1} \leqslant 1 / 2 ;(b) \bar{u}_{2} \equiv u_{2}[\phi] /\left.u_{2}[\phi]\right|_{x_{1}=1 / 2}$ near $x_{1}=1 / 2$. In $(b)$, open circles indicate the present numerical solution, while the solid lines indicate the curve of the least-squares approximation (3.7).

scheme, which actually constructs the Neumann series,

$$
\begin{gathered}
\psi_{n+1}=\int_{\mp 1 / 2}^{x_{1}} \frac{1}{k \zeta_{1}} \exp \left(-\frac{v(|\zeta|)}{k\left|\zeta_{1}\right|}\left|x_{1}-s\right|\right) K\left[\psi_{n}\right](s, \zeta) \mathrm{d} s, \quad \zeta_{1} \gtrless 0, \\
\psi_{0}=\phi_{0},
\end{gathered}
$$

$(n=0,1,2, \ldots)$ to obtain $\phi$ as a sum of the series $\phi=\sum_{i=0}^{\infty} \psi_{i}$. Because $\psi_{i} E^{1 / 2}$ is shown to be $O\left(k^{-i+1}(1+\ln k)^{i}\right)$, the practical convergence of the sum is fast for large $k$ (the rate of convergence is $O\left(k^{-1} \ln k\right)$ ). However, this method is not necessarily practical for the intermediate regime of $k$, because $\phi_{0}$ is not necessarily a good approximation of the solution. In the computations for the intermediate regime of $k$, it is better to start from an initial guess $\phi_{*}$ that is closer to the solution than $\phi_{0}$.

Let us denote by $\Psi_{*}$ the function that is obtained from (2.10a) by replacing $K[\phi]$ and $\phi_{0}$ with $K\left[\phi_{*}\right]$ and $\phi_{0}-\phi_{*}$ on its right-hand side:

$$
\Psi_{*}=\int_{\mp 1 / 2}^{x_{1}} \frac{1}{k \zeta_{1}} \exp \left(-\frac{v(|\zeta|)}{k\left|\zeta_{1}\right|}\left|x_{1}-s\right|\right) K\left[\phi_{*}\right](s, \zeta) \mathrm{d} s+\phi_{0}-\phi_{*}, \quad \zeta_{1} \gtrless 0 .
$$

Subtracting (3.5) from (2.10a) yields

$$
\phi-\phi_{*}=\Psi_{*}+\int_{\mp 1 / 2}^{x_{1}} \frac{1}{k \zeta_{1}} \exp \left(-\frac{v(|\zeta|)}{k\left|\zeta \zeta_{1}\right|}\left|x_{1}-s\right|\right) K\left[\phi-\phi_{*}\right](s, \zeta) \mathrm{d} s, \quad \zeta_{1} \gtrless 0 .
$$

Therefore, the solution is obtained as $\phi=\phi_{*}+\sum_{n=0}^{\infty} \psi_{n}$, as far as the scheme (3.4) with $\psi_{0}=\Psi_{*}$ gives a convergent sum $\sum_{n=0}^{\infty} \psi_{n}$. Note that in the above argument $\phi_{*}$ can be chosen freely. Actually we prepared $\phi_{*}$ that was expected to be closer than $\phi_{0}$ to the solution by preliminary calculations described in appendix A.

In the present work, we first carried out numerical computations by using the numerical code developed by Takata \& Funagane (2011), after making the above modification on the initial guess. The results for $k=10,6,2,1$ and 0.6 are shown in figure 2. Figure 2(a) shows the flow velocity profile in the half range $\left(0 \leqslant x_{1} \leqslant 1 / 2\right)$ with a solid line, while figure $2(b)$ shows the flow velocity near the boundary normalized by its value on the boundary with open circles. In figure $2(b)$ the 


$\begin{array}{lcccc}k & a & b & c & \text { Interval of } x_{1} \\ 10 & 0.3468 & -0.1640 & 0.1304 & {[0.499,0.5]} \\ 6 & 0.2940 & -0.1728 & 0.1196 & {[0.499,0.5]} \\ 2 & 0.1907 & -0.2003 & 0.0603 & {[0.4999,0.5]} \\ 1 & 0.1337 & -0.2221 & -0.0159 & {[0.4999,0.5]} \\ 0.6 & 0.0969 & -0.2378 & -0.106 & {[0.49998,0.5]}\end{array}$

TABLE 1. Coefficients of the fitting curve (3.7) for $u_{2}[\phi]$ near the upper boundary determined by the method of least squares using the data in the interval in the right-most column.

$\begin{array}{lcccccccc}\text { Interval of } & k=10 & k=6 & \begin{array}{c}\text { Interval of } \\ x_{1}\end{array} & k=2 & k=1 & \begin{array}{c}\text { Interval of } \\ x_{1}\end{array} & k=0.6 \\ {[0.496,0.5]} & -0.1639 & -0.1725 & {[0.4996,0.5]} & -0.2001 & -0.2216 & {[0.49995,0.5]} & -0.2377 \\ {[0.497,0.5]} & -0.1640 & -0.1726 & {[0.4997,0.5]} & -0.2002 & -0.2218 & {[0.49996,0.5]} & -0.2377 \\ {[0.498,0.5]} & -0.1640 & -0.1727 & {[0.4998,0.5]} & -0.2003 & -0.2219 & {[0.49997,0.5]} & -0.2377 \\ {[0.499,0.5]} & -0.1640 & -0.1728 & {[0.4999,0.5]} & -0.2003 & -0.2220 & {[0.49998,0.5]} & -0.2378\end{array}$

TABLE 2. Dependence of the coefficient $b$ in (3.7) on the choice of the interval of $x_{1}$ for the method of least squares.

curve of the least-squares approximation

$$
a+b\left(1 / 2-x_{1}\right) \ln \left(1 / 2-x_{1}\right)+c\left(1 / 2-x_{1}\right)
$$

with the values of $a, b$ and $c$ given in table 1 is also shown. The curve fits quite well to the original data on the grid points. The values of the coefficients $a, b$ and $c$ are almost independent of the choice of the interval in table 2. This shows the appearance of $\left(x_{1}-1 / 2\right) \ln \left|x_{1}-1 / 2\right|$ in the flow velocity near the boundary and the logarithmic divergence of its gradient.

Incidentally, as is seen from figure $2(a)$, the flow velocity differs between $k=6$ and 10. As $k$ is increased further, the flow velocity of the thermal transpiration between two parallel plates does not converge but rather grows logarithmically in $k$ (see, e.g., Niimi 1971; Chen et al. 2007). Such behaviour in the highly rarefied regime has also been studied by Takata \& Funagane (2011), where the main feature in the highly rarefied regime is shown to have already appeared at $k=10$. Indeed, the solution for $k=10$ was obtained only by seven iterations from $\phi_{0}$ using (3.4). Further details of the thermal transpiration in the highly rarefied regime can be found in Takata \& Funagane (2011).

\subsection{Numerical analysis: improvement of consistency}

In the numerical computations in $\S 3.2, K\left[\psi_{n}\right]$ is approximated by piecewise quadratic functions in $s$ to perform the integration with respect to $s$ in $(3.4 a)$. This is the way of approximation used by Takata \& Funagane (2011), which we call Method 1 for convenience. We first briefly discuss a possible weakness of this method for the present purpose and then improve it by using a more suitable approximation.

For illustrative purposes, let us consider the BGK model. Because of $K[\phi](s)=$ $2 \zeta_{2} u_{2}[\phi](s)$ and the discussions in $\S 3.2, K[\phi]$ behaves like $(s-1 / 2) \ln |s-1 / 2|$ near the boundary in this case. Therefore, the piecewise approximation in terms of quadratic 


\begin{tabular}{|c|c|c|c|c|c|c|c|}
\hline \multirow[b]{2}{*}{$k$} & \multicolumn{3}{|c|}{ Method 1} & \multicolumn{3}{|c|}{ Method 2} & \multirow[b]{2}{*}{ Interval of $x_{1}$} \\
\hline & $a$ & $b$ & $c$ & $a$ & $b$ & $c$ & \\
\hline 10 & 0.34681 & -0.16402 & 0.13044 & 0.34681 & -0.16402 & 0.13044 & {$[0.499,0.5]$} \\
\hline 6 & 0.29404 & -0.17279 & 0.11964 & 0.29404 & -0.17279 & 0.11964 & {$[0.499,0.5]$} \\
\hline 2 & 0.19075 & -0.20033 & 0.06032 & 0.19075 & -0.20033 & 0.06032 & {$[0.4999,0.5]$} \\
\hline 1 & 0.13371 & -0.22205 & -0.01594 & 0.13371 & -0.22205 & -0.01594 & {$[0.4999,0.5]$} \\
\hline 0.6 & 0.09689 & -0.23778 & -0.10639 & 0.09689 & -0.23778 & -0.10638 & {$[0.49998,0.5]$} \\
\hline
\end{tabular}

TABLE 3. Comparisons of the coefficients of the fitting curve (3.7) between Methods 1 and 2 , based on the data in the interval in the right-most column.

functions $P_{2}(s)$ is not consistent with the singular behaviour near the boundary and thus should be less accurate there than in the bulk region. The same singular feature is expected for $K[\phi]$ of a hard-sphere gas, because $K[\phi]$ is also a weighted integral of $\phi$.

In order to make the solution method consistent and to perform the computation more accurately, we newly approximate $K[\phi](s ; \zeta)$ by $\zeta_{2} P_{2}\left(s ; \zeta_{1},|\zeta|\right)+\zeta_{2} b\left(\zeta_{1},|\zeta|\right)(s \mp$ $1 / 2) \ln |s \mp 1 / 2|$ near the boundary in the numerical integration with respect to $s$, recalling the fact that $K[\phi] / \zeta_{2}$ is a function of three variables $s, \zeta_{1}$ and $|\zeta|$ because $K$ is axially symmetric. We call this approximation Method 2. Table 3 compares the coefficients of the curve of the least-squares approximation (3.7) determined by the results of Methods 1 and 2. The coefficients in both cases are almost identical, and thus we have reached the same conclusion as that in $\S 3.2$ in terms of the consistent method (Method 2). The difference between Methods 1 and 2 will be important in the discussions of another singularity at the microscopic level in $\S 4$.

In the meantime, in the case of the BGK model, the spatial singularity of $K[\phi]$ can be shown not to induce stronger spatial singularities in macroscopic quantities. Since there is no difference between the results obtained by using Methods 1 and 2, we can expect the same for the Boltzmann equation (not only for a hard-sphere gas) and have the following conjecture.

Conjecture 1. There are no spatial singularities contained in $K[\phi]\left(x_{1}\right)$ stronger than $\left(x_{1} \mp 1 / 2\right) \ln \left|x_{1} \mp 1 / 2\right|$ near $x_{1}= \pm 1 / 2$.

Further numerical results to be presented in $\S 4$ will make this statement more convincing.

To summarize, we have confirmed by the new consistent method that the spatial singularity $\left(x_{1} \mp 1 / 2\right) \ln \left|x_{1} \mp 1 / 2\right|$ appears in the flow velocity $u_{2}[\phi]$.

\section{Microscopic singularity on the boundary}

Figure 3 shows the velocity distribution function on the boundary at $x_{1}=1 / 2$ for $k=6,1$ and 0.6 that is obtained by Method 2. In addition to the widely known discontinuity at $\zeta_{1}=0$ (the molecular velocity parallel to the boundary), there is a common feature in the change of the VDF on the side of incident molecular velocity, namely a rather large slope at $\zeta_{1} \sim 0_{+}$that leads to the local maximum near there. We discuss this feature in the present section. 


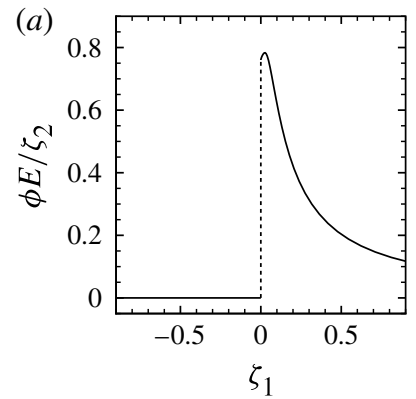

(b)

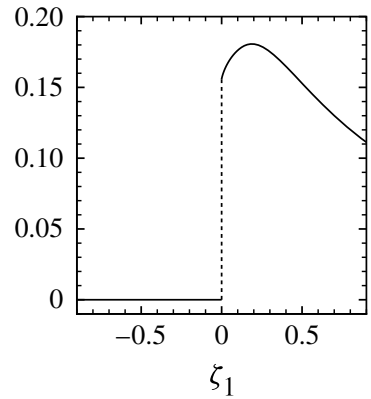

(c)

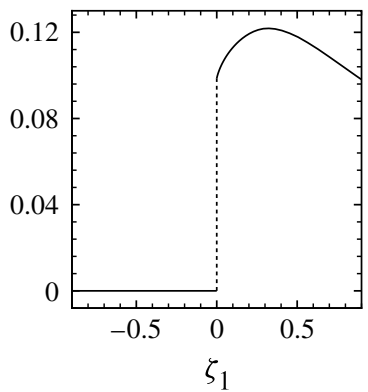

FIGURE 3. Velocity distribution function $\phi E / \zeta_{2}$ on the upper boundary $\left(x_{1}=1 / 2\right)$ for $|\zeta|=1.0:(a) k=6 ;(b) k=1 ;(c) k=0.6$.

Thanks to the symmetry of the problem, we consider only the upper boundary $x_{1}=1 / 2$, on which the formal solution $(2.10 a)$ is written as

$$
\phi= \begin{cases}\int_{-1 / 2}^{1 / 2} \frac{1}{k \zeta_{1}} \exp \left(-\frac{v}{k \zeta_{1}}\left(\frac{1}{2}-s\right)\right) K[\phi](s, \zeta) \mathrm{d} s+\phi_{0}, & \zeta_{1}>0 \\ 0, & \zeta_{1}<0\end{cases}
$$

Because $\phi_{0}=-(k / v)\left[1-\exp \left(-v / k \zeta_{1}\right)\right] I$ for $\zeta_{1}>0$, the derivative $\partial_{\zeta_{1}}\left(\phi_{0} / \zeta_{2}\right)$ (or $\partial_{\mu}\left(\phi_{0} / \zeta_{2}\right)$ ) vanishes at $\zeta_{1}=0_{+}$(or $\mu=0_{+}$) and thus is not related to the present concern. Let us examine the integral part.

In the case of the BGK model, $K[\phi]$ is regular in $\zeta$. Inspired by this and some numerical evidence, we assume that $K[\phi]$ is in general a regular function of $\zeta$ in the individual regions $\zeta_{1} \lessgtr 0$ and write it in the form

$$
\begin{aligned}
K[\phi]= & \left(\zeta_{2} /|\zeta|\right)\left[f_{-}\left(\zeta_{1},|\zeta|\right)\left(s-\frac{1}{2}\right) \ln \left|s-\frac{1}{2}\right|+f_{+}\left(\zeta_{1},|\zeta|\right)\left(s+\frac{1}{2}\right) \ln \left(s+\frac{1}{2}\right)\right. \\
& \left.+f_{0}\left(\zeta_{1},|\zeta|\right)+\sum_{m=1}^{\infty} s^{m} f_{m}\left(\zeta_{1},|\zeta|\right)\right]
\end{aligned}
$$

Here $f_{ \pm}, f_{0}$ and $f_{m}$ are regular in $\zeta_{1}$ and $|\zeta|$. Figure 4 shows a numerical example that supports the regular behaviour of $K[\phi]$ in $\zeta$. (Another example for a highly rarefied gas can be found in figure 6 of Takata $\&$ Funagane (2011). Moreover, the discussions in appendix B ensure that the conclusion in the present section does not change even if $K[\phi]$ behaves like $\zeta_{1} \ln \left|\zeta_{1}\right|$ near $\zeta_{1}=0$.) We examine the contribution term by term. Since the term of $f_{0}$ is independent of $s$, its contribution is essentially the same as that of $\phi_{0}\left(\phi_{0}\right.$ is the term representing the contribution from $\left.I\right)$ and thus changes moderately with respect to $\zeta_{1}$. The integrations of the remaining terms are reduced to

$$
\begin{gathered}
\int_{-1 / 2}^{1 / 2} \frac{1}{k \zeta_{1}} \exp \left(-\frac{v}{k \zeta_{1}}\left(\frac{1}{2}-s\right)\right)\left(\frac{1}{2}-s\right) \ln \left(\frac{1}{2}-s\right) \mathrm{d} s \\
=\frac{k \zeta_{1}}{v^{2}}\left(1-\gamma-\ln \frac{v}{k \zeta_{1}}-\exp \left(-\frac{v}{k \zeta_{1}}\right)-\operatorname{Ei}\left(1, \frac{v}{k \zeta_{1}}\right)\right),
\end{gathered}
$$


(a)

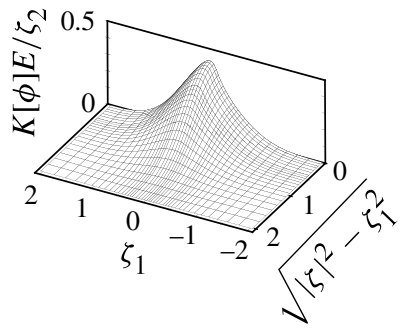

(b)

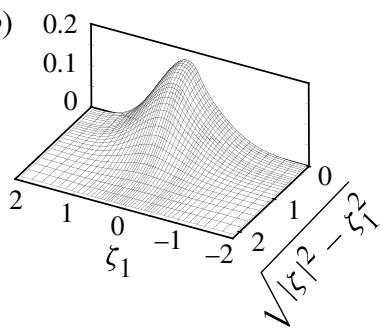

(c)

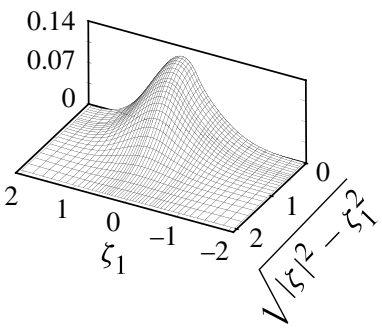

FIGURE 4. Plots of $K[\phi] E / \zeta_{2}$ on the boundary at $x_{1}=1 / 2$ for $(a) k=6,(b) k=1$ and $(c)$ $k=0.6$.

$$
\begin{gathered}
\int_{-1 / 2}^{1 / 2} \frac{1}{k \zeta_{1}} \exp \left(-\frac{v}{k \zeta_{1}}\left(\frac{1}{2}-s\right)\right)\left(\frac{1}{2}+s\right) \ln \left(\frac{1}{2}+s\right) \mathrm{d} s \\
=\frac{k \zeta_{1}}{v^{2}}\left(1-\gamma-\ln \frac{v}{k \zeta_{1}}-\exp \left(\frac{v}{k \zeta_{1}}\right)+\operatorname{Ei}\left(\frac{v}{k \zeta_{1}}\right)\right) \exp \left(-\frac{v}{k \zeta_{1}}\right), \\
\int_{-1 / 2}^{1 / 2} \frac{1}{k \zeta_{1}} \exp \left(-\frac{v}{k \zeta_{1}}\left(\frac{1}{2}-s\right)\right) s^{m} \mathrm{~d} s \\
=\frac{1}{2^{m}} \frac{1}{v}+(-1)^{m} \frac{1}{v}\left(\frac{k \zeta_{1}}{v}\right)^{m} \exp \left(-\frac{1}{2} \frac{v}{k \zeta_{1}}\right)\left(m \Gamma\left(m,-\frac{1}{2} \frac{v}{k \zeta_{1}}\right)-\Gamma(m+1)\right) \\
+\frac{(-1)^{m}}{m+1} \frac{1}{v}\left(\frac{k \zeta_{1}}{2 v}\right)^{m / 2} \exp \left(-\frac{3}{4} \frac{v}{k \zeta_{1}}\right) \mathrm{M}_{m / 2,(m+1) / 2}\left(\frac{1}{2} \frac{v}{k \zeta_{1}}\right),
\end{gathered}
$$

where $\mathbf{M}_{k, \mu}(z)$ is the Whittaker $\mathrm{M}$ function and Ei is the exponential integral defined on the real axis except the origin:

$$
\operatorname{Ei}(x)= \begin{cases}\text { p.v. } \int_{-\infty}^{x} t^{-1} e^{t} \mathrm{~d} t, & x>0, \\ -\operatorname{Ei}(1,-x), & x<0 .\end{cases}
$$

Equations (4.4) and (4.5) show that the integrals on the left-hand side change moderately with respect to $\zeta_{1}$. On the other hand, the third term in the parentheses of (4.3) induces a term proportional to $\zeta_{1} \ln \zeta_{1}$. Therefore, we conclude that the VDF changes steeply near $\zeta_{1}=0_{+}$and its derivative $\partial_{\zeta_{1}}\left(\phi / \zeta_{2}\right)\left(\right.$ or $\left.\partial_{\mu}\left(\phi / \zeta_{2}\right)\right)$ diverges logarithmically in the limit $\zeta_{1} \downarrow 0$. This gradient divergence at the microscopic level originates from (4.3) and thus from the spatial singularity $(s-1 / 2) \ln |s-1 / 2|$ of $K[\phi]$.

In order to reinforce the above discussions, we show in figure 5 the numerical solution $\phi$ for incident molecules $\left(\zeta_{1}>0\right)$ near $\zeta_{1}=0$ on the boundary at $x_{1}=1 / 2$ that is obtained by Methods 1 and 2 for different grid systems. Since Method 1 approximates $K[\phi]$ by piecewise quadratic functions in $s$, it cannot capture the concerned singularity $\zeta_{1} \ln \left|\zeta_{1}\right|$ in principle. Due to this fact, the convergence of the numerical solution with respect to the spatial grid size is slow in Method 1. In contrast to Method 1, the convergence of the solution by Method 2 is quite satisfactory. Moreover, the profile is almost linear in $\zeta_{1} \ln \left|\zeta_{1}\right|$, which also supports the conclusion in the previous paragraph.

We here give brief information on the grid systems that we used. In the coarse grid, $\mathrm{C}$, the grid size is uniformly 0.05 in the half channel $[0,0.5]$. In the intermediate 

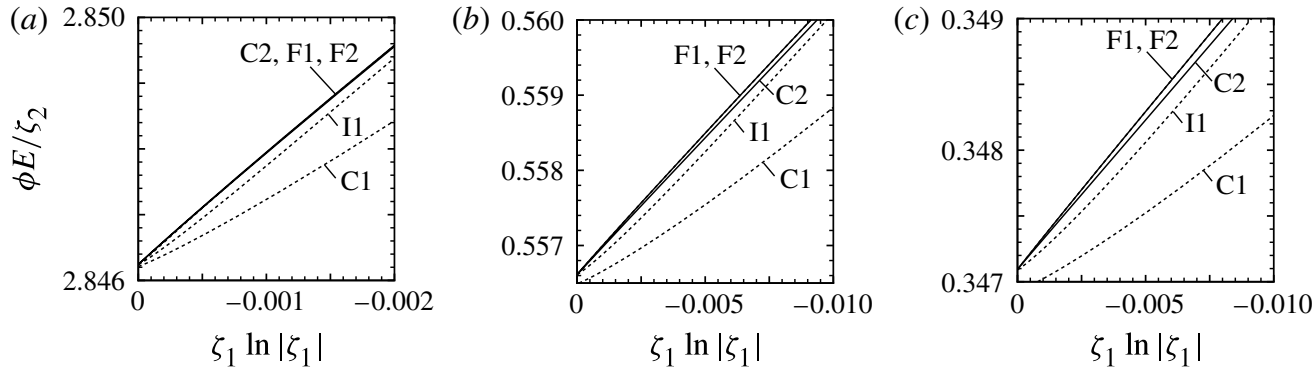

FIGURE 5. Comparisons of $\phi E / \zeta_{2}$ between Methods 1 and 2 on the upper boundary $\left(x_{1}=1 / 2\right)$ for small $\zeta_{1}>0$ and $|\zeta|=0.5$ : (a) $k=6 ;$ (b) $k=1 ;(c) k=0.6$. Solid line: Method 2; dashed line: Method 1. The labels C1 (or C2), I1 and F1 (or F2) represent the results of Method 1 (or 2) obtained by the spatially coarse, intermediate and fine grid systems, respectively. Note that the difference between F1 and F2 (among F1, F2 and C2 in (a)) is invisible in the figure.

grid, I, the grid size is 0.05 in $[0,0.45]$ and 0.005 in $[0.45,0.5]$. In the fine grid, $\mathrm{F}$, the half channel is divided into three parts [0, 0.45], [0.45, 0.495], [0.495, 0.5] for $k=6$, four parts [0, 0.45], [0.45, 0.495], [0.495, 0.4995], [0.4995, 0.5] for $k=1$ and five parts [0, 0.45], [0.45, 0.495], [0.495, 0.4995], [0.4995, 0.49995], [0.49995, 0.5] for $k=0.6$; and the grid size in each part is proportional to the width of the part. For example, the grid size is $0.05,0.005$ and 0.0005 for $k=6$ in the first, second and third parts, respectively. The grid was required to be finer near the boundary for smaller $k$, because the kinetic-theory (or non-fluid-dynamical) structure in the present concern tends to be more localized near the boundary as $k$ becomes smaller.

To summarize, it is shown that the spatial singularity $\left(x_{1} \mp 1 / 2\right) \ln \left|x_{1} \mp 1 / 2\right|$ of $K[\phi]$ induces a singularity $\zeta_{1} \ln \left|\zeta_{1}\right|$ of the VDF on the boundary $x_{1}= \pm 1 / 2$. Thus, there is a logarithmic divergence of its derivative with respect to $\zeta_{1}$ at $\zeta_{1}=0_{ \pm}$at $x_{1}= \pm 1 / 2$. The rapid convergence of the numerical solution by Method 2 with respect to the spatial grid size further supports Conjecture 1 in $\S 3.3$.

\section{Spatial singularity and the discontinuity of the VDF}

In the present section, we will show that the spatial singularity of macroscopic quantities investigated in $\S 3$ can be related quantitatively to the discontinuity of the velocity distribution function on the boundary. We will show this quantitative correspondence through a simple damping model.

As in $\S 4$, we consider only the upper boundary $x_{1}=1 / 2$ and denote by $\epsilon$ the distance from it, i.e. $\epsilon=1 / 2-x_{1}$. As far as $K[\phi]$ is regular in $\zeta$ as is assumed in (4.2), its part for $\zeta_{1}>0$ does not contribute to the spatial singularity of $u_{2}[\phi]$. Indeed,

$$
\begin{aligned}
& \left.\partial_{x_{1}} \int_{0}^{1} \int_{-1 / 2}^{x_{1}} \frac{1}{k \zeta \mu} \exp \left(-\frac{v(\zeta)}{k \zeta \mu}\left(x_{1}-s\right)\right) \mathrm{d} s \mathrm{~d} \mu\right|_{x_{1}=1 / 2}=\frac{1}{k \zeta} \operatorname{Ei}\left(1, \frac{v(\zeta)}{k \zeta}\right) \\
& \left.\partial_{x_{1}} \int_{0}^{1} \int_{-1 / 2}^{x_{1}} \frac{1}{k \zeta \mu} \exp \left(-\frac{v(\zeta)}{k \zeta \mu}\left(x_{1}-s\right)\right)\left(\frac{1}{2}-s\right) \ln \left(\frac{1}{2}-s\right) \mathrm{d} s \mathrm{~d} \mu\right|_{x_{1}=1 / 2} \\
& \quad=\frac{1}{v(\zeta)}\left(\gamma+\ln \frac{v(\zeta)}{k \zeta}+\operatorname{Ei}\left(1, \frac{v(\zeta)}{k \zeta}\right)\right)
\end{aligned}
$$


42

\section{S. Takata and H. Funagane}

$$
\begin{aligned}
& \left.\partial_{x_{1}} \int_{0}^{1} \int_{-1 / 2}^{x_{1}} \frac{1}{k \zeta \mu} \exp \left(-\frac{v(\zeta)}{k \zeta \mu}\left(x_{1}-s\right)\right)\left(\frac{1}{2}-s\right) \mathrm{d} s \mathrm{~d} \mu\right|_{x_{1}=1 / 2} \\
& \quad=\frac{1}{v(\zeta)}\left(-1+\exp \left(-\frac{v(\zeta)}{k \zeta}\right)\right),
\end{aligned}
$$

and the integrals of the above right-hand sides multiplied by a regular function of $\zeta$ do not diverge on the boundary. On the other hand, for $\zeta_{1}<0$,

$$
\begin{gathered}
\int_{0}^{1} \int_{1 / 2}^{x_{1}} \frac{1}{k \zeta \mu} \exp \left(-\frac{v(\zeta)}{k \zeta \mu}\left(s-x_{1}\right)\right) \mathrm{d} s \mathrm{~d} \mu \\
=-\epsilon \int_{0}^{1} \int_{0}^{1} \frac{1}{k \zeta \mu} \exp \left(-\frac{v(\zeta) \epsilon}{k \zeta \mu} s\right) \mathrm{d} s \mathrm{~d} \mu \\
=-\frac{1}{v(\zeta)}\left(1-\exp \left(-\frac{v(\zeta) \epsilon}{k \zeta}\right)+\frac{v(\zeta) \epsilon}{k \zeta} \operatorname{Ei}\left(1, \frac{v(\zeta) \epsilon}{k \zeta}\right)\right), \\
\int_{0}^{1} \int_{1 / 2}^{x_{1}} \frac{1}{k \zeta \mu} \exp \left(-\frac{v(\zeta)}{k \zeta \mu}\left(s-x_{1}\right)\right)\left(\frac{1}{2}-s\right) \ln \left(\frac{1}{2}-s\right) \mathrm{d} s \mathrm{~d} \mu \\
=-\epsilon^{2} \int_{0}^{1} \int_{0}^{1} \frac{1}{k \zeta \mu} \exp \left(-\frac{v(\zeta) \epsilon}{k \zeta \mu} s\right)(1-s)(\ln \epsilon+\ln (1-s)) \mathrm{d} s \mathrm{~d} \mu \\
=O\left((\epsilon \ln \epsilon)^{2}\right), \\
\int_{0}^{1} \int_{1 / 2}^{x_{1}} \frac{1}{k \zeta \mu} \exp \left(-\frac{v(\zeta)}{k \zeta \mu}\left(s-x_{1}\right)\right)\left(\frac{1}{2}-s\right) \mathrm{d} s \mathrm{~d} \mu \\
=-\epsilon^{2} \int_{0}^{1} \int_{0}^{1} \frac{1}{k \zeta \mu} \exp \left(-\frac{v(\zeta) \epsilon}{k \zeta \mu} s\right)(1-s) \mathrm{d} s \mathrm{~d} \mu=O\left(\epsilon^{2} \ln \epsilon\right) .
\end{gathered}
$$

Therefore, only the value of $K[\phi]$ on the boundary contributes to $-(\epsilon / k \zeta) \operatorname{Ei}(1, v \epsilon / k \zeta)$ (the third term of the furthest right-hand side of the first equation), giving rise to the concerned spatial singularity. Moreover, the integrals of the above integrands multiplied by $\mu, \mu^{2}, \ldots$ do not induce the spatial singularity. Therefore, the part of $K[\phi]$ that changes around $\zeta_{1}=0$ with respect to $\zeta_{1}$ does not contribute to the spatial singularity.

In the long run, the contribution of $K[\phi]$ to the spatial singularity $\left(x_{1} \mp 1 / 2\right) \ln (1 / 2 \mp$ $x_{1}$ ) is limited to its value at $x_{1}= \pm 1 / 2$ and $\zeta_{1}=0$. Because the contribution from $I$, namely $\phi_{0}$, induces the spatial singularity as shown in $\S 3.1$, the concerned spatial singularity of $u_{2}[\phi]$ should be recovered exactly as the corresponding moment $u_{2}[f]$ of

$$
f=-\left.\frac{k}{v}\left(\frac{1}{k} K[\phi]-I\right)\right|_{\zeta_{1}=0, x_{1}= \pm 1 / 2} \exp \left(-\frac{v}{k \zeta_{1}}\left(x_{1} \mp \frac{1}{2}\right)\right), \quad \zeta_{1} \lessgtr 0 .
$$

Equation (5.7) is the solution of a simple damping model

$$
\begin{gathered}
\zeta_{1} \frac{\partial f}{\partial x_{1}}=-\frac{v}{k} f, \\
f=-\left.\frac{k}{v}\left(\frac{1}{k} K[\phi]-I\right)\right|_{\zeta_{1}=0, x_{1}= \pm 1 / 2}, \quad \zeta_{1} \lessgtr 0, x_{1}= \pm 1 / 2 .
\end{gathered}
$$




\begin{tabular}{lccc} 
& \multicolumn{2}{c}{$b$} & \\
\cline { 2 - 3 }$k$ & $u_{2}[\phi]$ & $u_{2}[f]$ & Interval of $x_{1}$ \\
10 & -0.1640 & -0.1641 & {$[0.499,0.5]$} \\
6 & -0.1728 & -0.1730 & {$[0.499,0.5]$} \\
2 & -0.2003 & -0.2005 & {$[0.4999,0.5]$} \\
1 & -0.2221 & -0.2223 & {$[0.4999,0.5]$} \\
0.6 & -0.2378 & -0.2379 & {$[0.49998,0.5]$}
\end{tabular}

TABLE 4. Comparisons of the coefficient $b$ of the fitting curve (3.7) between $u_{2}[\phi]$ of the original problem and $u_{2}[f]$ of the damping model.

The boundary data in $(5.8 b)$ has a clear physical meaning, which we will now explain. Since $\phi$ is a solution of $(2.9 a)$, we have

$$
\phi\left(x_{1}, \zeta_{1}=0\right)=\left.\frac{k}{v}\left(\frac{1}{k} K[\phi]-I\right)\right|_{\zeta_{1}=0} \text { for }-1 / 2<x_{1}<1 / 2 .
$$

(The same expression can be obtained from $(2.10 a)$ by taking the limit $\zeta_{1} \rightarrow 0_{ \pm}$.) This expression gives the limiting value of $\phi$ for $\zeta_{1}=0_{ \pm}$and $x_{1} \rightarrow \pm 1 / 2$, and thus we have the following relation on the boundary

$$
\begin{aligned}
- & \left.\frac{k}{v}\left(\frac{1}{k} K[\phi]-I\right)\right|_{\zeta_{1}=0, x_{1}= \pm 1 / 2} \\
& =\phi\left(x_{1}= \pm 1 / 2, \zeta_{1}=0_{\mp}\right)-\phi\left(x_{1}= \pm 1 / 2, \zeta_{1}=0_{ \pm}\right) .
\end{aligned}
$$

This relation means that the boundary data in $(5.8 b)$ is no other than the discontinuity of the velocity distribution function at $\zeta_{1}=0$ on the boundary (see figure 3 ). Therefore, the spatial singularity of the macroscopic quantity is quantitatively related to the discontinuity of the VDF on the boundary. In other words, the macroscopic singularity is a trace of the discontinuity of the VDF on the boundary.

Table 4 shows comparisons between the coefficient $b$ of the curve of the leastsquares approximation for the numerical solution obtained by Method 2 and that for the solution of the damping model (5.8). The two results are almost identical and strongly support the conclusion in the present section.

\section{Conclusion}

We have discussed the appearance of singularity both at macroscopic and microscopic levels on a planar boundary by studying the thermal transpiration problem as a specific example. Although we assume a hard-sphere gas in the numerical investigation, the theoretical framework developed here relies only on four properties: (i) spatially one dimensional; (ii) the linearized collision integral can be split into two parts $-v \phi$ and $K[\phi]$; (iii) the (dimensionless) collision frequency $v$ is positive definite; (iv) $K$ has a smoothing effect and accordingly $K[\phi]$ does not behave singularly in $\zeta$. Therefore, the three main conclusions for the planar boundary, namely:

(a) macroscopic quantities behave like $x \ln x$ with respect to the distance $x$ from the planar boundary, and thus its spatial gradient normal to the boundary diverges logarithmically on the boundary; 
(b) velocity distribution function for the molecules incoming to the boundary behaves like $\zeta_{n} \ln \left|\zeta_{n}\right|$ for $\zeta_{n} \simeq 0$ on the boundary, and thus its derivative with respect to $\zeta_{n}$ also diverges logarithmically, where $\zeta_{n}$ is the normal component of the molecular velocity to the boundary;

(c) the macroscopic singularity $x \ln x$ is quantitatively related to the discontinuity of the velocity distribution function at $\zeta_{n}=0$ on the boundary;

apply also to gas models with a more general (cut-off) intermolecular potential such as Grad's hard potential.

It should be noted that the singularity studied in the present paper is not caused by the linearization of the problem. In fact, the identified source of the singularity, namely the discontinuity of the velocity distribution function on the planar boundary, usually exists irrespective of whether the system is linearized or not; thus, the damping model in $\S 5$ remains useful even for the nonlinear system. The difference is the fact that the collision frequency $v$ for the nonlinear system is not a known function of $\zeta$ but its function through the velocity distribution function. Roughly speaking, this $v$ is a local-density-like quantity (actually the local density in the case of the cut-off Maxwell molecule) and is expected to show the same macroscopic singularity as that discussed in the present paper. However, such a singularity part in $v$ does not contribute to the present structure of singularity. The singularity studied in the present paper should also be observed in the nonlinear Boltzmann system.

We make a brief remark on the case that the solution depends also on the spatial coordinates in the tangential direction $\left(x_{2}\right.$ and/or $\left.x_{3}\right)$ and on the case of a more general boundary condition. In the former case, a spatial integration of a function $\zeta_{n} \ln \left|\zeta_{n}\right|$ can appear in the formal solution, through a spatial derivative of the VDF with respect to $x_{2}$ and/or $x_{3}$ for incoming molecules to the boundary. In the latter case, especially in the case of Maxwell's boundary condition (a linear combination of the diffuse and specular reflections), the partial specular reflection can cause $\zeta_{n} \ln \left|\zeta_{n}\right|$ for outgoing molecules from the boundary and thus its spatial integration. Since more general boundary conditions are expressed in terms of a weighted integral of the VDF of incoming molecules, the case of Maxwell's condition is the worst case from the viewpoint of the strength of singularity. Thus, bearing in mind these observations and examining the influence of the $\zeta_{n} \ln \left|\zeta_{n}\right|$ term in the analysis, we see as shown in appendix B that the singularity $x \ln x$ (or a further severe singularity) is not induced by the spatial integration of $\zeta_{n} \ln \left|\zeta_{n}\right|$. Therefore, the main conclusions listed above apply to general situations on the planar boundary.

Finally, in the case of the specular reflection boundary, since the VDF is continuous on the boundary, the $x \ln x$ dependence should not appear in the macroscopic quantities, and accordingly the VDF should not show the $\zeta_{n} \ln \left|\zeta_{n}\right|$ dependence. Our theoretical conclusion of no spatial singularity on the planar specular-reflection boundary agrees with the results of the Knudsen-layer analyses for the BGK model by Sone \& Aoki (1977) (Aoki, private communication). A rigorous mathematical proof of the microscopic singularity (conclusion $(b)$ in the above list) for sufficiently large Knudsen numbers will be treated in a separate paper by Chen, Funagane, Liu \& Takata.

\section{Acknowledgements}

The present work has been partially supported by KAKENHI from JSPS (No. 23656068). The authors thank Professor K. Aoki in Kyoto University and Professor T.-P. Liu and Dr I.-K. Chen in Academia Sinica, Taipei for their interests and helpful discussions. The authors also thank $\mathrm{Mr} \mathrm{M}$. Oishi for his help in preliminary studies by using the BGK model. 


\section{Appendix A. Construction of the initial guess and the data of computations}

Let us denote by $\phi_{\#}$ the solution for $k=k_{\#}$ and by $h$ its difference from the solution

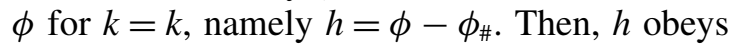

$$
\begin{gathered}
\zeta_{1} \frac{\partial h}{\partial x_{1}}=-\frac{1}{k} v h+\frac{1}{k} K[h]-\left(\frac{1}{k_{\#}}-\frac{1}{k}\right)\left(-v \phi_{\#}+K\left[\phi_{\#}\right]\right) \\
\left(=-\frac{1}{k} v h+\frac{1}{k} K[h]-\left(1-\frac{k_{\#}}{k}\right)\left(\zeta_{1} \frac{\partial \phi_{\#}}{\partial x_{1}}+I\right)\right), \\
\text { b.c. } h=0, \quad \zeta_{1} \lessgtr 0, x_{1}= \pm \frac{1}{2},
\end{gathered}
$$

and thus $\phi$ is expressed as $\phi=\phi_{\#}+\sum_{n=0}^{\infty} \psi_{n}$ in terms of the function series $\left\{\psi_{n}\right\}$ generated by $(3.4 a)$ by putting

$$
\psi_{0}=\left(1-\frac{k}{k_{\#}}\right) \int_{\mp 1 / 2}^{x_{1}} \exp \left(-\frac{v}{k\left|\zeta_{1}\right|}\left|x_{1}-s\right|\right) \frac{v}{k \zeta_{1}}\left(-\phi_{\#}+\frac{K\left[\phi_{\#}\right]}{v}\right) \mathrm{d} s, \quad \zeta_{1} \gtrless 0 .
$$

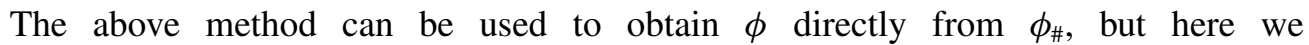
have used it for preparing the initial guess $\phi_{*}$ for the iterative solution process for $k<10$. The initial guess $\phi_{*}$ has been prepared by using the coarse spatial grid system (see the penultimate paragraph of $\S 4$ ) under a loose criteria of convergence. Actually, we have constructed the initial guess in the following order: $k=10 \rightarrow 8 \rightarrow 6 \rightarrow 4 \rightarrow 3 \rightarrow 2 \rightarrow 1 \rightarrow 0.8 \rightarrow 0.6$ by $7,8,9,11,12,19,15$ and 19 iterations, respectively (for instance, there are 15 iterations for preparing the initial data for $k=6$ from $k=10$ ). Although the required number of iterations is not small, the computational time can be effectively reduced, thanks to the coarse grid and loose convergence criteria. In this process, the refined interpolation for spatial integration, Method 2 described in $\$ 3.3$, was not used. After preparing the initial guess, the required number of iterations was 4,12 and 17 for $k=6,1$ and 0.6 in the case of the fine spatial grid system (F1 and F2).

The information of the spatial grid is given in the penultimate paragraph of $\S 4$. As in Takata \& Funagane (2011), we treated $\widetilde{\phi}=\phi / \zeta_{2}$ as a function of $\left(x_{1}, \zeta_{1}, \zeta_{\rho}\right)$, where $\zeta_{\rho}=\sqrt{|\xi|^{2}-\zeta_{1}^{2}}$, instead of $\left(x_{1}, \zeta_{1},|\xi|\right)$ in the actual numerical calculations and truncated the regions of $\zeta_{1}$ and $\zeta_{\rho}$ into $-4.6<\zeta_{1}<4.6$ and $0<\zeta_{\rho}<6.2$ for $k=0.6,-4.6<\zeta_{1}<4.6$ and $0<\zeta_{\rho}<5.2$ for $k=1$ and 2 , and $-7.4<\zeta_{1}<7.4$ and $0<\zeta_{\rho}<7.4$ for $k=6$ and 10. The number of grid points in $\zeta_{1}$ is 194 for $k=0.6,1,2$, 6 and 10, while that in $\zeta_{\rho}$ is 99 for $k=0.6$ and 97 for other cases.

As a measure of accuracy of our numerical solution, we have compared the data of mass flow rate for $k=0.6,1,2,6$ and 10 with the well-established data of Ohwada et al. (1989) and Kosuge et al. (2005) that have been obtained by the finite-difference method using hundreds of spatial grid points. Our results agree to four digits with Kosuge et al. (2005). The influence of the choice of spatial grid system is also small for the mass flow rate. Even when Method 1 is used with the coarse spatial grid, namely $\mathrm{C} 1$, the difference can appear only at the fourth decimal point by unity for the worst case $(k=0.6)$.

The computations have been carried out on a parallel computer system that is composed of Xeon Quad Core W3565 3.2 GHz. It takes about 22, 36 and 60 hours to obtain the solution for $k=6,1$ and 0.6 by Method 2 with the fine grid system (F2) using 32, 50 and 50 cores, respectively. With the coarse grid system (C2), 
the computational time is reduced to about one fifth to one quarter. There is almost no difference of the computational time between Methods 1 and 2.

\section{Appendix B. Influence of $\zeta_{1}\left|\ln \zeta_{1}\right|$ on the estimate of spatial singularity}

Consider the boundary $x_{1}=1 / 2$ and introduce the variable of distance $\epsilon=1 / 2-x_{1}$. If $K[\phi]$ has a singularity of $\zeta_{1} \ln \left|\zeta_{1}\right|$ for $\zeta_{1}>0$ (namely for the molecules incoming to the boundary), we see that

$$
\begin{aligned}
& \lim _{x_{1} \rightarrow 1 / 2} \partial_{x_{1}} \int_{0}^{1} \int_{-1 / 2}^{x_{1}} \frac{1}{k \zeta \mu} \mu \ln \mu \exp \left(-\frac{v(\zeta)}{k \zeta \mu}\left(x_{1}-s\right)\right) \mathrm{d} s \mathrm{~d} \mu \\
& =\lim _{x_{1} \rightarrow 1 / 2} \partial_{x_{1}} \int_{0}^{1} \frac{1}{v} \mu \ln \mu\left(1-\exp \left(-\frac{v(\zeta)}{k \zeta \mu}\left(x_{1}+\frac{1}{2}\right)\right)\right) \mathrm{d} \mu \\
& =\lim _{x_{1} \rightarrow 1 / 2} \int_{0}^{1} \frac{1}{k \zeta} \ln \mu \exp \left(-\frac{v(\zeta)}{k \zeta \mu}\left(x_{1}+\frac{1}{2}\right)\right) \mathrm{d} \mu \\
& =\frac{1}{v} \int_{0}^{1} \frac{v}{k \zeta \mu} \mu \ln \mu \exp \left(-\frac{v(\zeta)}{k \zeta \mu}\right) \mathrm{d} \mu .
\end{aligned}
$$

Because the furthest right-hand side is bounded by a constant, the spatial singularity is not induced by $\zeta_{1} \ln \left|\zeta_{1}\right|$ on the side of incoming molecules. On the other hand, for $\zeta_{1}<0$ (namely for the outgoing molecules), we have

$$
\begin{aligned}
& \int_{0}^{1} \int_{1 / 2}^{x_{1}} \frac{1}{k \zeta \mu} \mu \ln \mu \exp \left(-\frac{v(\zeta)}{k \zeta \mu}\left(s-x_{1}\right)\right) \mathrm{d} s \mathrm{~d} \mu \\
& =-\epsilon \int_{0}^{1} \int_{0}^{1} \frac{1}{k \zeta} \ln \mu \exp \left(-\frac{v(\zeta) \epsilon}{k \zeta \mu} s\right) \mathrm{d} s \mathrm{~d} \mu \\
& =-\frac{1}{v(\zeta)} \int_{0}^{1} \mu \ln \mu\left(1-\exp \left(-\frac{v(\zeta) \epsilon}{k \zeta \mu}\right)\right) \mathrm{d} \mu
\end{aligned}
$$

where the furthest right-hand side is evaluated as

$$
\begin{aligned}
(0 \leqslant) & -\int_{0}^{1} \mu \ln \mu\left(1-\exp \left(-\frac{v(\zeta) \epsilon}{k \zeta \mu}\right)\right) \mathrm{d} \mu \\
& \leqslant \int_{0}^{1} \sqrt{\mu}\left(1-\exp \left(-\frac{v(\zeta) \epsilon}{k \zeta \mu}\right)\right) \mathrm{d} \mu \\
= & \frac{4}{3} \sqrt{\pi}\left(\frac{v(\zeta) \epsilon}{k \zeta}\right)^{3 / 2}\left(\operatorname{erf}\left(\sqrt{\frac{v(\zeta) \epsilon}{k \zeta}}\right)-1\right) \\
& +\frac{2}{3}\left(1-\exp \left(-\frac{v(\zeta) \epsilon}{k \zeta}\right)+2 \frac{v(\zeta) \epsilon}{k \zeta} \exp \left(-\frac{v(\zeta) \epsilon}{k \zeta}\right)\right) .
\end{aligned}
$$

Now consider the integral of the furthest right-hand side of this estimate multiplied by $\zeta^{2} E(\zeta)$, bearing in mind the contribution from the Jacobian in the coordinate system $(\zeta, \mu, \varphi)$. The result is $O(\epsilon)$, which implies that the spatial singularity is not induced by $\zeta_{1} \ln \left|\zeta_{1}\right|$ on the side of the outgoing molecules. 


\section{REFERENCES}

Bhatnagar, P. L., Gross, E. P. \& Krook, M. 1954 A model for collision processes in gases. I. Small amplitude processes in charged and neutral one-component systems. Phys. Rev. 94, 511-525.

BIRD, G. A. 1994 Molecular Gas Dynamics and the Direct Simulation of Gas Flows. Oxford.

Chen, C.-C., Chen, I.-K., LiU, T.-P. \& Sone, Y. 2007 Thermal transpiration for the linearized Boltzmann equation. Commun. Pure Appl. Math. 60, 0147-0163.

ChEn, I.-K., LIU, T.-P. \& TAKATA, S. 2010 Boundary singularity for thermal transpiration problem of the linearized Boltzmann equation. Preprint, Institute of Mathematics, Academia Sinica (submitted to Arch. Rat. Mech. Anal.).

Holway, L. H. Jr 1963 Approximation procedures for kinetic theory. PhD thesis, Harvard University.

Holway, L. H. Jr 1966 New statistical models for kinetic theory: methods of construction. Phys. Fluids 9, 1658-1673.

Kennard, E. H. 1938 Kinetic Theory of Gases. McGraw-Hill.

Kosuge, S., Sato, K., TAKata, S. \& Aoki, K. 2005 Flows of a binary mixture of rarefied gases between two parallel plates. In Rarefied Gas Dynamics (ed. M. Capitelli), pp. 150-155. American Institute of Physics.

LILlEy, C. R. \& SADER, J. E. 2007 Velocity gradient singularity and structure of the velocity profile in the Knudsen layer according to the Boltzmann equation. Phys. Rev. E 76, 026315.

Lilley, C. R. \& SAder, J. E. 2008 Velocity profile in the Knudsen layer according to the Boltzmann equation. Proc. R. Soc. A 464, 2015-2035.

LOYALKA, S. K. 1971 Kinetic theory of thermal transpiration and mechanocaloric effect. I. J. Chem. Phys. 55, 4497-4503.

MAXWELl, J. C. 1879 On stresses in rarefied gases arising from inequalities of temperature. Phil. Trans. R. Soc. 170, 231-256.

NiImI, H. 1971 Thermal creep flow of rarefied gas between two parallel plates. J. Phys. Soc. Jpn 30, 572-574.

Ohwada, T., Sone, Y. \& Aoki, K. 1989 Numerical analysis of the Poiseuille and thermal transpiration flows between two parallel plates on the basis of the Boltzmann equation for hard-sphere molecules. Phys. Fluids A 1, 2042-2049.

Sone, Y. 1964 Kinetic theory analysis of linearized Rayleigh problem. J. Phys. Soc. Jpn 19, 1463-1473.

Sone, Y. 1965 Effect of sudden change of wall temperature in a rarefied gas. J. Phys. Soc. Jpn 20, 222-229.

Sone, Y. 1966 Thermal creep in rarefied gas. J. Phys. Soc. Jpn 21, 1836-1837.

Sone, Y. 2007 Molecular Gas Dynamics. Birkhäuser.

SONE, Y. \& AOKI, K. 1977 Slightly rarefied gas flow over a specularly reflecting body. Phys. Fluids 20, 571-576.

SONE, Y. \& ONISHI, Y. 1978 Kinetic theory of evaporation and condensation - Hydrodynamic equation and slip boundary condition. J. Phys. Soc. Jpn 44, 1981-1994.

TAKATA, S. \& Funagane, H. 2011 Poiseuille and thermal transpiration flows of a highly rarefied gas: over-concentration in the velocity distribution function. J. Fluid Mech. 669, 242-259.

Welander, P. 1954 On the temperature jump in a rarefied gas. Ark. Fys. 7, 507-553.

Williams, M. M. R. 1971 Boundary-value problems in the kinetic theory of gases. Part 2. Thermal creep. J. Fluid Mech. 45, 759-768. 